\title{
Association vs dissociation and setting appropriate criteria for object agnosia
}

\author{
Lúcia Garrido $^{\mathrm{a}}$, Bradley Duchaine ${ }^{\mathrm{b}}$, and Joseph DeGutis ${ }^{\mathrm{c}}$ \\ aDivision of Psychology, Brunel University London, Uxbridge, Middlesex, UK \\ ${ }^{b}$ Psychological and Brain Sciences, Dartmouth College, Hanover, NH, USA \\ 'Department of Psychiatry, Harvard Medical School, VA Boston Healthcare System, Boston, MA, \\ USA
}

\begin{abstract}
We are grateful to Geskin and Behrmann (2017) for carrying out their exhaustive review and, in doing so, bringing the issues discussed in the review and the accompanying commentaries to the fore. Twenty years ago, only a handful of congenital prosopagnosia (CP) cases had been reported, so the number of cases in the review is a testament to the rapid growth of $\mathrm{CP}$ research. The review is motivated by a question that received attention even in the earliest CP research (McConachie, 1976): what is the relationship between face and object recognition? Their review indicates that around $80 \%$ of CPs tested have deficits with object recognition, whereas about $20 \%$ have face-specific deficits. These figures led the authors to suggest that face and object recognition are likely to depend on a common mechanism and that face-specific mechanisms are unlikely to exist. In our commentary, we discuss two points that challenge this conclusion. First, because the inferential value of cases showing a dissociation between two abilities is greater than the value of cases showing an association between the same two abilities, we feel the substantial proportion of CPs with face-specific deficits actually provides support for a model where face and object recognition depend on different processes. Second, we believe that the criteria used to classify participants as impaired with objects are too liberal, and we show below that they can lead to misidentification of object deficits even in a substantial proportion of a control sample.
\end{abstract}

\section{Association, dissociation, and the relationship between face recognition and object recognition}

Demonstrations that neuropsychological participants have impairments in different types of tasks (associations) can be revealing (Caramazza, Miceli, \& Villa, 1987), but when tasks matched for difficulty and task demands show association in some participants and dissociation in other participants, standard neuropsychological logic would suggest that the two tasks involve, at least in part, different mechanisms. Evidence of association is often difficult to interpret, because in patients with acquired brain damage, lesions may have

Disclosure statement

No potential conflict of interest was reported by the authors. 
affected multiple processes, while in participants with developmental deficits, the developmental processes at fault may have impacted the functioning of different mechanisms. Most brain damaged patients with prosopagnosia also have object agnosia (Barton, 2008; Damasio, Damasio, \& Van Hoesen, 1982), but several cases have shown convincing dissociations between impaired face recognition and spared object recognition (Busigny, Graf, Mayer, \& Rossion, 2010; Rezlescu et al., 2012; Sergent \& Signoret, 1992). Conversely, Mr. C.K. recognized upright faces normally but was severely impaired with objects (Moscovitch, Winocur, \& Behrmann, 1997). Together, these findings from braindamaged patients indicate that face and object recognition depend on neighbouring processes that can dissociate from each other. Selective impairments involving transcranial magnetic stimulation (Pitcher, Charles, Devlin, Walsh, \& Duchaine, 2009) and intracranial stimulation (Schalk et al., 2017) also support this view.

Geskin and Behrmann's (2017) review indicates that, like acquired prosopagnosia, many CPs have object agnosia, but around 20\% have normal object recognition. While we think the $20 \%$ figure should be treated cautiously due to the limited testing done with many CPs, the double-dipping problem mentioned in the review, and questions about $\mathrm{CP}$ categorization that we discuss below, we interpret this estimate as evidence against the notion that face and object recognition depend on the same processes. In our view, the associations between prosopagnosia and object agnosia in the CPs are challenging to interpret because they may result from the atypical development of neighbouring brain mechanisms, and, given that these mechanisms are likely to depend on shared developmental processes (Ramus, 2004), we expect associations to be frequent, just like in acquired prosopagnosia. The dissociations, however, provide much clearer evidence about cognitive organization. Geskin and Behrmann (2017), on the other hand, stated they consider the associations in CPs to be more revealing than the dissociations, and they favour the common mechanism account because "a theory based on explaining the distribution of all the data will likely provide the best account of the phenomenon" (p. 42). We find this statement puzzling, because the authors do not explain how the common mechanism explanation can account for spared object recognition in CP. Moreover, the inferential limitations of evidence of association are well known in neuropsychology (Shallice, 1988), and thus the proposal that face and object recognition depend on different mechanisms is consistent with the frequent co-occurrence of prosopagnosia and object agnosia.

\section{The criteria to categorize CPs as object agnosic are far too liberal}

In addition to these theoretical issues, we also think Geskin and Behrmann's (2017) criteria for classifying object agnosia in CPs are far too lenient and will lead to many false positives. Regardless of how many object tests a CP scored normally on, the authors classified a prosopagnosic as having a definite object recognition deficit if that person had at least one score on an object recognition test that was two or more standard deviations below the control mean, and a mild object recognition deficit if they had at least one score that was between 1.7 and 2.0 standard deviations below the mean. These criteria are particularly problematic for the many cases in which multiple tests of object recognition were used (see Appendices in Geskin \& Behrmann, 2017). For example, EB/Edward scored normally on 21 of 23 measures of non-face recognition even without counting his normal accuracy and RTs 
throughout greeble training (Duchaine et al., 2006), but he was categorized as object agnosic (see Appendix 5 in Geskin \& Behrmann, 2017) because his accuracy with scenes was just outside the normal range, and his RT for horses was $>2$ standard deviations above the mean. Similar performance on face identity tests would obviously not suggest prosopagnosia.

The likelihood of having abnormal performance on at least one test increases with the number of tests and also depends on their correlation (Crawford, Garthwaite, \& Gault, 2007). To further illustrate this point, we used the exact same criteria as Geskin and Behrmann (2017) to compare how many individuals from the control sample in Garrido et al. (2009) (CPs in the review were compared to these controls) would be classified as having object agnosia. This sample included 18 controls matched to $17 \mathrm{CPs}$, and all control and CP participants did the same behavioural battery. Here, we considered performance on the four object recognition tests used by Geskin and Behrmann (2017) to classify our CPs. These tests consisted of two old-new recognition tests (cars, horses), and two matching tasks (bodies, novel objects). We collected accuracy and response times for these tests, resulting in eight dependent measures. Using the same criteria as Geskin and Behrmann (2017), six of our 18 controls (i.e., 33\%) would be classified as having a definite object recognition deficit, and three $(17 \%)$ controls would be classified as having a mild object recognition deficit. Therefore, $50 \%$ of our control sample would be classified as having object agnosia. This result shows how common it is to have at least one abnormal score when analysing eight measures, and it serves as a point of comparison to the estimate that $76 \%$ of our group of $\mathrm{CPs}^{1}$ had at least one abnormal score in an object recognition test. Our control sample consisted of young ( $M_{\text {age }}=28.94$ years, $S D=5.70$, range $\left.=23-43\right)$, well-educated individuals with a mean IQ of $118.94(S D=8.75$, range $=103-133)$, and none complained of object recognition difficulties. It is inconceivable that $50 \%$ of them have object agnosia, and we would be surprised if even $5 \%$ are object agnosic.

\section{Future investigations of object processing in CP}

Going forward, we believe the use of a number of practices will provide the field with more confidence about individual CP's object processing abilities. First, multiple object tests should be used, and, when doing so, it is best to estimate the percentage of people in the healthy population that have one (or more) impaired scores and to then compare the performance of individual cases with those base percentages to find out how abnormal they are (Crawford et al., 2007). Those base percentages can be calculated empirically with data from a large control sample or estimated using Monte Carlo simulations (Crawford et al., 2007). Second, future studies would also benefit from using more extensively validated and normed measures. In particular, future studies should focus on measures that have been sufficiently validated to reflect individual differences in object discrimination/recognition specifically, rather than more general cognitive abilities like speed of processing or general intelligence (Richler, Wilmer, \& Gauthier, 2017). Additionally, when using RT measures from traditional accuracy tasks, it is important to include instructions to respond as quickly

\footnotetext{
1. Geskin and Behrmann (2017) misclassified a few of our cases and included some of our cases more than once in the Appendices. When we reclassified our $17 \mathrm{CP}$ cases according to their criteria using the eight dependent measures described above, 13 (76\%) would be classified as having definite object recognition deficit, 0 would have mild object recognition deficit, and 4 would have "no deficit" (DP4, DP10, DP11, and DP12).
} 
and accurately as possible (e.g., several tests in the meta-analysis did not include instructions about speed of response). Also, when using RTs from tasks with prolonged/self-paced stimulus presentation, it can be difficult to distinguish excessive carefulness from impairment. Thus, when possible, stimuli should be shown for a limited duration, so longer RTs provide minimal benefit. Further, it would be advantageous to use measures with sufficiently large and diverse normative samples $(N>100)$, where adjustments for age and other demographic factors are possible. This is particularly relevant because in the current review, several CPs are classified as having object processing deficits in studies where the age of the control group is younger than that of the CP group (e.g., Huis in't Veld, Van den Stock, \& de Gelder,2012; Righart \& de Gelder, 2007). Although we acknowledge that these recommendations are a high bar, we hope that as the field of CP research grows, it can adopt some of these methodological improvements in order to better inform this important debate.

\section{References}

Barton JJS (2008). Structure and function in acquired proso-pagnosia: Lessons from a series of 10 patients with brain damage. Journal of Neuropsychology, 2, 197-225. [PubMed: 19334311]

Busigny T, Graf M, Mayer E, \& Rossion B (2010). Acquired prosopagnosia as a face-specific disorder: Ruling out the general visual similarity account. Neuropsychologia, 48, 2051-2067. [PubMed: 20362595]

Caramazza A, Miceli G, \& Villa G (1987). The role of the graphemic buffer in spelling: Evidence from a case of acquired dysgraphia. Cognition, 26, 59-85. [PubMed: 3608396]

Crawford J, Garthwaite P, \& Gault C (2007). Estimating the percentage of the population with abnormally low scores (or abnormally large score differences) on standardized neuropsychological test batteries: A generic method with applications. Neuropsychology, 21, 419-430. [PubMed: 17605575]

Damasio A, Damasio H, \& Van Hoesen G (1982). Prosopagnosia: Anatomic basis and behavioral mechanisms. Neurology, 32, 331-341. [PubMed: 7199655]

Duchaine B, Yovel G, Butterworth E, \& Nakayama K (2006). Prosopagnosia as animpairment to facespecific mechanisms: Elimination of the alternative hypotheses in a developmental case. Cognitive Neuropsychology, 23, 714-747. [PubMed: 21049351]

Garrido L, Furl N, Draganski B, Weiskopf N, Stevens J, Tan G,... Duchaine B (2009). Voxel-based morphometry reveals reduced grey matter volume in the temporal cortex of developmental prosopagnosics. Brain, 132, 3443-3455. [PubMed: 19887506]

Geskin J, \& Behrmann M (2017). Congenital prosopagnosia without object agnosia? A literature review. Cognitive Neuropsychology 1-51. doi:10.1080/02643294.2017.1392295

Huis in't Veld E., Van den Stock J, \& de Gelder B (2012). Configuration perception and face memory, and face context effects in developmental prosopagnosia. Cognitive Neuropsychology, 29(5-6), 464-481. [PubMed: 23190402]

McConachie H (1976). Developmental prosopagnosia. A single case report. Cortex, 12, 76-82. [PubMed: 1261287]

Moscovitch M, Winocur G, \& Behrmann M (1997). What Is special about face recognition? Nineteen experiments on a person with visual object agnosia and dyslexia but normal face recognition. Journal of Cognitive Neuroscience, 9,555-604. [PubMed: 23965118]

Pitcher D, Charles L, Devlin JT, Walsh V, \& Duchaine B (2009). Triple dissociation of faces, bodies, and objects in extrastriate cortex. Current Biology, 19, 319-324. [PubMed: 19200723]

Ramus F (2004). Neurobiology of dyslexia: A reinterpretation of the data. Trends in Neurosciences, 27, 720-726. [PubMed: 15541512]

Rezlescu C, Pitcher D, \& Duchaine B (2012). Acquired proso-pagnosia with spared within-class object recognition but impaired recognition of degraded basic-level objects. Cognitive Neuropsychology, 29, 325-347. [PubMed: 23216309] 
Richler JJ, Wilmer JB, \& Gauthier I (2017). General object recognition is specific: Evidence from novel and familiar objects. Cognition, 166, 42-55. [PubMed: 28554084]

Righart R, \& de Gelder B (2007). Impaired face and body perception in developmental prosopagnosia. Proceedings of the National Academy of Sciences, 104(43), 17234-17238.

Schalk G, Kapeller C, Guger C, Ogawa H, Hiroshima S, Lafer-Sousa R,... Kanwisher N (2017). Facephenes and rainbows: Causal evidence for functional and anatomical specificity of face and color processing in the human brain. Proceedings of the National Academy of Sciences, 114, $12285-12290$.

Sergent J, \&Signoret JL (1992). Varieties of functional deficits in prosopagnosia. Cerebral Cortex, 2, 375-388. [PubMed: 1422092]

Shallice T (1988). From neuropsychology to mental structure. Cambridge: Cambridge University Press. 